\title{
Investigating the Source of Contamination Opioid into Lead in Forensic Medicine
}

\section{Seyed Majid Salimi AsI ${ }^{1 *}$, Mohammad Assareh ${ }^{1}$, Mohammad Javad Khodayar ${ }^{2}$ and Zahra Mousavi ${ }^{3}$}

${ }^{1}$ Department of Forensic Toxicology, Forensic Research Center, Forensic Medicine Organization, Tehran, Iran

${ }^{2}$ Department of Toxicology, School of Pharmacy, Ahvaz Jundishapur University of Medical Sciences, Ahvaz, Iran

${ }^{3}$ Department of Pharmacology-Toxicology, Faculty of Pharmacy, Pharmaceutical Sciences Branch, Islamic Azad University, Tehran, Iran

${ }^{*}$ Corresponding author: Salimi Asl SM, Department of Forensic Toxicology, Forensic Research Center, Forensic Medicine Organization, Tehran, Iran, Tel: +986132210815; E-mail: majid.salimi2012@yahoo.com

Received date: November 13, 2017; Accepted date: December 18, 2017; Published date: December 22,2017

Copyright: (C) 2017 Salimi Asl SM, et al. This is an open-access article distributed under the terms of the Creative Commons Attribution License, which permits unrestricted use, distribution, and reproduction in any medium, provided the original author and source are credited.

Citation: Salimi AsI SM, Assareh M, Khodayar MJ, Mousavi Z (2017) Investigating the Source of Contamination Opioid into Lead in Forensic Medicine. J Heavy Met Toxicity Dis. Vol. 2 No. 2: 4.

\section{Abstract}

Background: Impurities in narcotics are one of the factors that cause or exacerbate the symptoms of acute and chronic poisoning. Heavy metals as one of the impurities found in the opioids can cause toxicity to consumers. Opioids contamination and its complications are a social health problem in Middle Eastern countries such as Iran. Lead smugglers add more benefits during the drug production process in order to increase their weight.

Objective: To determine the source of opioid contamination with lead metal.

Materials and methods: Preparation of Opioid, Heroin, and Crock Afghan Drugs for quantitative analysis is used for Wet Digestion (oxidation or wet digestion), for analysis of a flame atomic spectrometer. Air-acetylene flame gas, halogen-based lamp based on the metal studied.

Results: LOD and LOQ for lead, 2 PPM and 6 PPM spectroscopy were determined for flame atomic absorption spectrometry.

Conclusion: According to the results, the level of lead in the poppy basin is negative as a source of opium production. However, lead was found in opium, burned opium, cracker Afghani and heroin as opium products. Which suggests that lead in the production of opium and its natural and industrial opioids is likely to be added to the benefits of smugglers and vendors.

Keywords: Opioid; Lead; Forensic medicine; Atomic Absorption Spectroscopy

\section{Introduction}

Impurities in the drug can cause or exacerbate the symptoms of acute and chronic toxicity of these substances. For example, lead as one of the impurities found in narcotics can cause poisoning [1]. Drug abuse and its complications are a social health problem in Middle Eastern countries such as Iran [2]. Smugglers may add lead during narcotics production, which is more in favor of weight gain, so that the pattern of the presence of impurities in the same drug (chemical fingerprint) can reflect the geographic location of its production [3]. In addition, many non-specific symptoms of drug dependence show signs of lead poisoning [4-8].

Drug overdose is one of the major causes of mortality from poisoning in our country [9-12]. Deaths from acute drug poisoning are important in many aspects of medicine, in particular forensic medicine. Although the diagnosis of respiratory system syndrome and its complications are one of the major causes of mortality in acute intoxication among drug users However, the presence of possible impurities in consumers can lead to the emergence and aggravation of signs and symptoms of poisoning. Therefore, identifying and quantifying the potential impurities, both for the treatment of clinical toxicology and for the Determining the cause of death is important in legal toxicology [1]. An important category of this impurity is heavy metals such as lead and arsenic, which in some cases are an important component of impurities in opium and heroin $[13,14]$.

The purpose of this paper is to determine the source of contamination of opium and its derivatives to lead heavy metal.

\section{Materials and Methods}

Lead Acetate, Reagents for Chlorhexaplatin, Acid Neutritic from the German Merck Company. Distilled water distilled twice, TLC plate from MN. 


\section{Sample collection and preparation}

The drugs were delivered to the forensic laboratory, and then the samples were weighed and reported in terms of color, shape, solid or powder, and.... After this, the nature of the narcotics should be determined by the use of TLC as opium, heroin, and Afghan cracker.

After separation of samples for preparation of samples for quantitative analysis, wet digestion method was used [9]. The $20 \mathrm{mg}$ portion of the weighed sample was shaken up in a Chinese capsule with $2 \mathrm{ml}$ of nitric acid (35\% v/v) with a glass rod until complete digestion, and then the contents of the Chinese capsule were transferred to a $50 \mathrm{ml}$ balloon and distilled water was added to the volume. It should be noted that all glass units are washed with nitric acid (10\% v/v) and then distilled twice with distilled water to remove any possible contamination from metals.

First, a standard curve was prepared and validated. To draw the standard curve by an atomic-flame absorption spectrometer, distilled water was first distilled twice and $2.5 \%$ nitric acid was used as a blank. Then, absorption of standard solutions the lead was read by GBC Avanta.

Note: Absorbance of each sample, including standard blanks, samples, was repeated at least 3 times in all experiments, and repeated if changes were more than $5 \%$.

The standard curve was plotted in terms of Absorption versus concentration (in PPM).

The minimum concentration of standard solution and the signal-to-noise ratio were used to determine the detectable amount and the minimum measurable amount of the sequential dilution method. To prepare the standard solution, dissolved juice was dissolved in distilled water and distilled twice in a $100 \mathrm{ml}$ balloon. Then, by continuous dilution with distilled water, two distillates from the standard stock were prepared with concentrations of 10, 20, 30, 40, 50 PPM. Then, in the steps described, it is injected by an atomic-flame absorption spectrometer in front of the halogenated metal bulb and the results are reported by the stability.

\section{Results}

Based on LOD and LOQ results for copper, 2 PPM and 6 PPM are used for flame atomic absorption spectroscopy. The lead concentration graph is in the range of 0-50 PPM linear Graph (Figure 1). Based on the results, the level of lead in the poppy basin is negative as a source of opium production.

But lead was found in opium, burnt opium, Afghan crackles and heroin as opium derivatives (Figure 2), indicating that lead has been added to the production of opium and natural and industrial alkaloids, which are likely to be added to the benefits of smugglers and sellers.

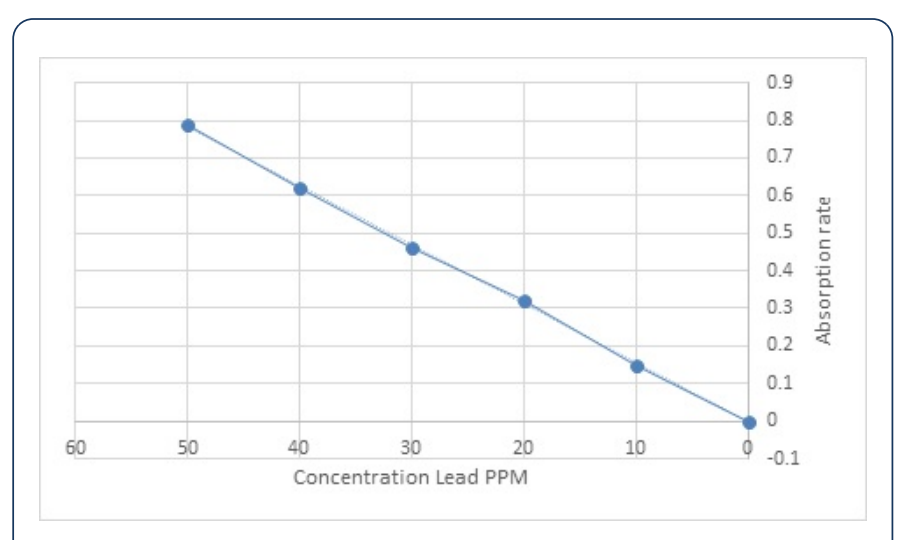

Figure 1 Standard lead concentration and absorption.

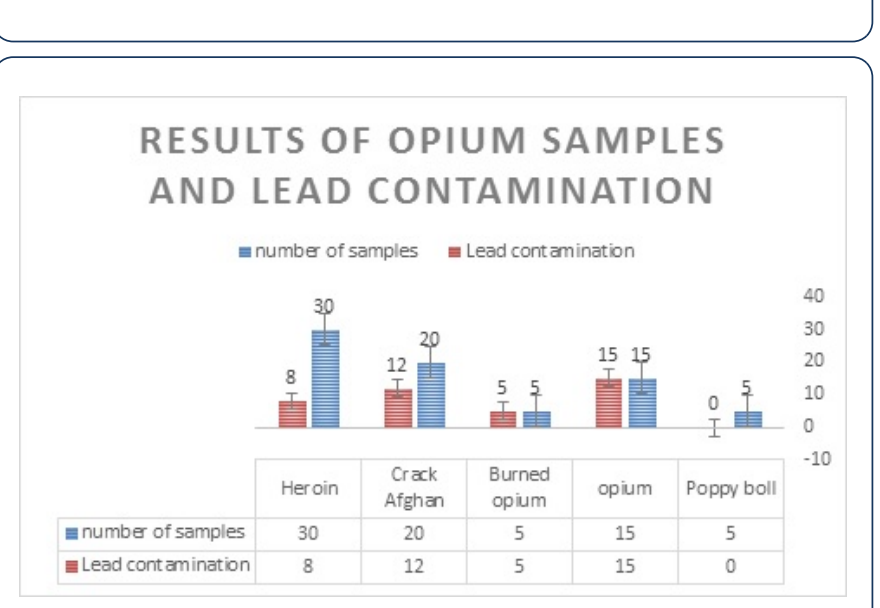

Figure 2 Opium sample and lead contamination.

\section{Discussion}

The intrinsic properties of drugs are important in terms of toxicity and clinical significance due to their complications. The most important impurities in the opium are sand, pebbles, clay, ash, starch, starches and heavy metals such as arsenic $[8,9]$. Among the various methods of detecting and detecting heavy metals such as colorimetric, voltammetry of anodic strip, X-ray fluorescence spectroscopy And atomic spectrometry for biological and non-biological samples, the flame atomic spectrometry method has the accuracy, sensitivity and ease [10].

Based on clinical and clinical studies in Iran's hospitals, the main complications of opium poisoning and its derivatives are related to lead metal contamination. Opium addicts treatment has been shown to be less effective, regardless of lead poisoning treatment, and to treat and detoxify the patient's lead. The results have been satisfactory. Based on the findings of Dr. Fatemi and colleagues, a 25-year-old man came across complaints of abdominal pain and progressive nausea and vomiting from about 2 months ago and $7 \mathrm{~kg}$ of weight loss during this period. The patient was the one who suffered from generalized bone pain, especially back pain and jaundice in the eyes. The patient had a long history of oral and oral intake. The physical examination of jaundice and mucus was evident and was observed in the examination of the black pigmentation in 
the margin area of the gum. Hepatosplenomegaly and lymphatic anodipathy were not observed. The upper gastrointestinal tract was reported in the endoscopic examination. In the experiments, the liver aminotransferases increased, but alkaline phosphates was normal. The indirect bilirubin also increased. In the blood test, non-immune anemia, hemolytic anemia was reported and direct and indirect coombs were negative. The BMB and BMA showed an erythroid hyperplasia, and the patient presented a history of smoking and opium from about 6 years ago. With regard to a series of symptoms, with suspicion of lead poisoning, the level of lead in the blood was measured, with a level of $350 \mathrm{mg} / \mathrm{dl}$ reported to be a very high level of lead poisoning. At the same time, high levels of drug abuse were reported. The patient was treated with calcium EDTA, B.A.L, and the symptoms of the patient improved with a gradual decrease in blood lead levels. Similar cases of disease due to oral drug use that have been reported in lead studies have been reported in Iran [15].

The purpose of this paper is to find the source of contamination of opium addicts and derivatives to lead metal. By exploring poppy cultivation as the primary source of opium production and its family, we concluded that lead contamination after poppy cultivation by opium smugglers for weight gain and more profits are being made, causing acute and chronic complications in drug addicts.

\section{Conflict of Interest}

In this paper, there was no conflict with the legal and ethical interests.

\section{Funding and Support}

The cost of this study was personal and academic support was received from the Department of Toxicology, Pharmaceutical Sciences Branch, Islamic Azad University, Tehran, Iran.

\section{Acknowledgments}

Thanks to the professors and colleagues who helped me in this article.

\section{References}

1. Soltani NK, Faryadi M, Akhri M (2004) Determination of lead in opium by flame atomic absorption spectroscopy. Scientific J Forensic Med 9: 176-179.
2. US Department of Health and Human Services (1999) Agency for Toxic Substances and Disease Registry. Toxicological Profile for Lead. Atlanta: GA, Public Health Service 587.

3. Kaa E, Bent K (1986) Impurities, adulterants and diluents of illicit heroin in Denmark (Jutland and Funen). Forensic Sci Int 31: 195-210.

4. Chia BL, Leng CK, Hsii FP, Yap MH, Lee YK (1973) Lead poisoning from contaminated opium. Br Med J 1: 354.

5. Beattie AD, Mullin PJ, Baxter RH, Moore MR (1979) Acute lead poisoning: an unusual cause of hepatitis. Scott Med J 24: 318-321.

6. Fitzsimons EJ, Dagg JH (1982) Lead poisoning in a drug addict; the intravenous injection of suppository extracts. Br J Clin Pract 36: $284-285$.

7. Parras F, Patier JL, Ezpeleta C (1987) Lead-contaminated heroin as a source of inorganic-lead intoxication. N Engl J Med 316: 755.

8. D'Alessandro-Gandolfo L, Macri A, Biolcati G, Rossi F, Cirelli A, et al. (1989) An unusual mechanism of lead poisoning. Presentation of a case. Recenti Prog Med 80: 140-141.

9. Bermejo-Barrera $P$, Moreda-Piñeiro $A$, Moreda-Piñeiro J, Bermejo-Barrera A, Bermejo-Barrera AM (1999) "A study of illicit cocaine seizure classification by pattern recognition techniques applied to metal data." J Forensic Sci 44: 270-274.

10. Aqa Bayloubi Abbas, Peyvand Abdolkarim, Afshar Mahshid (1381) Acute Complications and Causes of Death in Patients Admitted to ICU Poisoning at Loghman Hakim Hospital in Tehran in the Second Six Months of 2000. Research in Medical Sciences. 1: 1 .

11. Kazemian Mohammad, Vatan Doost Hassan, Mousavi Shahram, Hassanian Hassan. Epidemiologic characteristics of adult poisoning during the year 2000 in Loghman Hakim Hospital in Tehran. Research in Medical Sciences. 21: 1-18.

12. Fares Nagari, Pejomand Abdolkarim, Nazervar Bashir. The Indicator, a Reflection of the First Six Months of Death in 2000, Referral to the National Forensic Medicine Organization. J Forensic Med. 27: 27-22.

13. Narang AP, Chawla LS, Khurana SB (1987) Levels of arsenic in Indian opium eaters. Drug Alcohol Depend Nov 20: 149-153.

14. Bora T, Merdivan M, Hamamci C (2002) Levels of trace and major elements in illicit heroin. J Forensic Sci 47: 959-963.

15. Fatemi Sayed R, Jafarzadeh F, Mostafa E, Zali Mohammad R (2009) Scientific J Medical Council of the Islamic Republic of Iran: Spring Volume 27: 117-120. 